Check for updates

Cite this: RSC Adv., 2017, 7, 30495

\title{
A facile procedure to modify filter paper for oil- water separation
}

\author{
Xinde Tang, (D)* Chunli Shen, Wenjing Zhu, Shuai Zhang, Youfu Xu, Yueqing Yang, \\ Min Gao and Fuying Dong
}

A much simpler and broadly applicable dip-coating method using silica nanoparticles and a silane coupler for preparing hydrophobic paper is presented. Both producing surface hydrophobization without additional surface treatment and increasing the adhesive force between the paper and the coated material can be realized by an almost one-step procedure. The modified filter paper could not only selectively permeate oil from a mixture, but also exhibits superior recyclability, indicating its great potential application in the oil-water separation area.

Received 1st April 2017

Accepted 24th May 2017

DOI: 10.1039/c7ra03754f

rsc.li/rsc-advances

deformation and loss of permeation. Such paper is usually

\section{Introduction}

Oil leakage and industrial wastewater discharge, not only resulting in the loss of precious resources but also threatening the environment and human health, have attracted considerable attention during the past decades. ${ }^{\mathbf{1 , 2}}$ Oil-water separation is extensively applied in the areas of petroleum, chemicals, and water treatment. ${ }^{3}$ Since the separation process is related to interfacial issues, to design materials with special wettability is an effective and facile way by either filtration or absorption of a single phase selectively., ${ }^{4,5}$ So far various approaches, such as photolithography, ${ }^{6}$ electrodeposition, ${ }^{7}$ colloidal assembly, ${ }^{8}$ chemical vapor deposition, ${ }^{9}$ and electro-spinning, ${ }^{10}$ have been developed to construct materials with special surface wettability. Specifically, superhydrophobic surfaces have been employed for effective oil-water separation. ${ }^{11-16}$ However, these approaches usually suffer from some disadvantages including complicated and time-consuming fabrication processes, high cost and low efficiency, which restrict the practical and commercial applications. ${ }^{17}$ Therefore, developing simple, scalable, and cost-effective methods are highly desirable.

Paper, composed of fibrous cellulose, as a ubiquitous and essential materials, has been widely used to fabricate composite materials with specific functions. Paper-based materials and devices for microfluidic, ${ }^{18}$ electronics, ${ }^{19}$ sensing $^{20}$ and separation ${ }^{21,22}$ have drawn extensive attention due to their benefits with regards to light weight, low cost, environmental friendliness, ease of fabrication, and the wide scope of applications. In particular, the paper with special wettability has been applied in surface protection, ${ }^{23}$ longterm conservation, ${ }^{24}$ and oil/water separation. ${ }^{21,22}$ Among them, superhydrophobic paper plays an important role in practical applications, whereas faces the challenges such as

School of Material Science and Engineering, Shandong Jiaotong University, Jinan 250357, China.E-mail: xdtang8033@163.com prepared by modification of nanoparticle with hydrophobic material, resulting in hierarchical structures with low surface energy and high roughness. Ogihara et al. reported a simple method for preparing superhydrophobic paper with high water-repellency and transparency by spray-deposited hydrophobic silica nanoparticle coatings. ${ }^{23}$ Our group developed one-step dip coating process to fabricate a transparent superhydrophobic coatings on the surface of paper using hexadecyltrimethoxysilane-modified silica nanoparticles. ${ }^{24} \mathrm{Lu}$ et al. prepared superhydrophobic and superoleophilic filter paper for separating liquids with low surface tensions such as oil and ethanol from water by treating commercially available filter paper with a mixture of hydrophobic silica nanoparticles and polystyrene solution in toluene. ${ }^{21}$ Above-mentioned approaches introduce simpler processes, however, they involved in the hydrophobic modification of nanoparticle before the fabrication of superhydrophobic paper. Therefore, trying to further simplify process is desired.

In this paper, we present a much simpler and broadly applicable dip-coating method using silica nanoparticles and a silane coupler for the preparation of highly hydrophobic paper. This method does not require additional surface treatment by tedious and time-consuming processes to increase the adhesive force between the paper and the coated material, or to produce surface hydrophobization, which is an almost one-step procedure.

\section{Experimental}

\section{Materials}

Fumed silica nanoparticle with diameter of $40-60 \mathrm{~nm}$ was kindly offered by Prof. Feng's group in Shandong University of China. The silane coupler hexadecyltrimethoxysilane (HDTMS) 
was purchased from Aladdin Reagent Company in China. Whatman filter paper was purchased from Jingdong Mall in China. The other reagents were used as received without further purification.

\section{Preparation of modified filter paper}

In a $100 \mathrm{~mL}$ beaker, a certain amount of silica nanoparticle was dispersed in the mixture of anhydrous ethanol $(9.0 \mathrm{~mL})$, deionized water $(1.0 \mathrm{~mL})$, HDTMS $(0.5 \mathrm{~mL})$, and triethylamine (0.5 $\mathrm{mL}$ ) under ultrasonication for $20 \mathrm{~min}$, resulting in a dispersion solution with silica content ranging from 2.0 to 4.0 wt\%. Dip-coating method was employed to modify filter paper. Specifically, a piece of common filter paper was immersed into as-prepared dispersion solution under ultrasonication for $10 \mathrm{~s}$, then taken out and dried at ambient temperature overnight.

\section{Preparation and separation of oil-water mixtures and emulsions}

Three types of oil-water mixtures were obtained by mixing oil and deionized water (1:1 and $95: 5, \mathrm{v} / \mathrm{v})$ under ultrasonication for $30 \mathrm{~min}$, which contained different oil components such as petroleum ether (b.p. $60-90{ }^{\circ} \mathrm{C}$ ), dichloromethane, and toluene. The SDS-stabilized water-in-oil emulsions were prepared by dissolving $0.04 \mathrm{mg} \mathrm{mL}^{-1}$ of sodium dodecyl sulfate (SDS) in oilwater mixturers $(95: 5, \mathrm{v} / \mathrm{v})$ under same mixing conditions. All these mixtures and emulsions stayed stable before separation experiments without stratification and precipitation.

Separation experiments for oil-water mixtures and emulsions were conducted through modified filter paper under the action of gravity separation. To evaluate the wettability and durability of the modified filter paper, the separation device maintained the original state for further observing the residual liquid in filter paper after complete oil-water separation, and recycling the modified filter paper was conducted.

\section{Characterization}

The surface morphologies of the original and modified filter paper were observed by scanning electron microscope (SEM, Hitachi S-4800) at $8 \mathrm{kV}$. The surface wettability of filter paper was evaluated by water contact angle (WCA) measurement (CA100A) equipped with a high-speed camera, the droplet size of deionized water was controlled to be $5 \mu \mathrm{L}$. The surface composition of coating was measured by X-ray photoelectron spectroscopy (XPS, PHI-5300), full scan with step of $1 \mathrm{eV}$ and pass energy of $89.45 \mathrm{eV}$, as well as narrow scan with step of $0.2 \mathrm{eV}$ and pass energy of $35.75 \mathrm{eV}$. Dynamic light scattering (DLS, Zetasizer Nano ZS90) was performed to characterize the droplet size. The optical microscopy images were obtained by Optical microscope (Axio Scope.A1).

\section{Results and discussion}

\section{Fabrication of modified filter paper}

Various approaches have been applied to fabricate superhydrophobic surface for oil-water separation. ${ }^{5,21}$ In this paper, a facile procedure to modify filter paper for oil-water separation

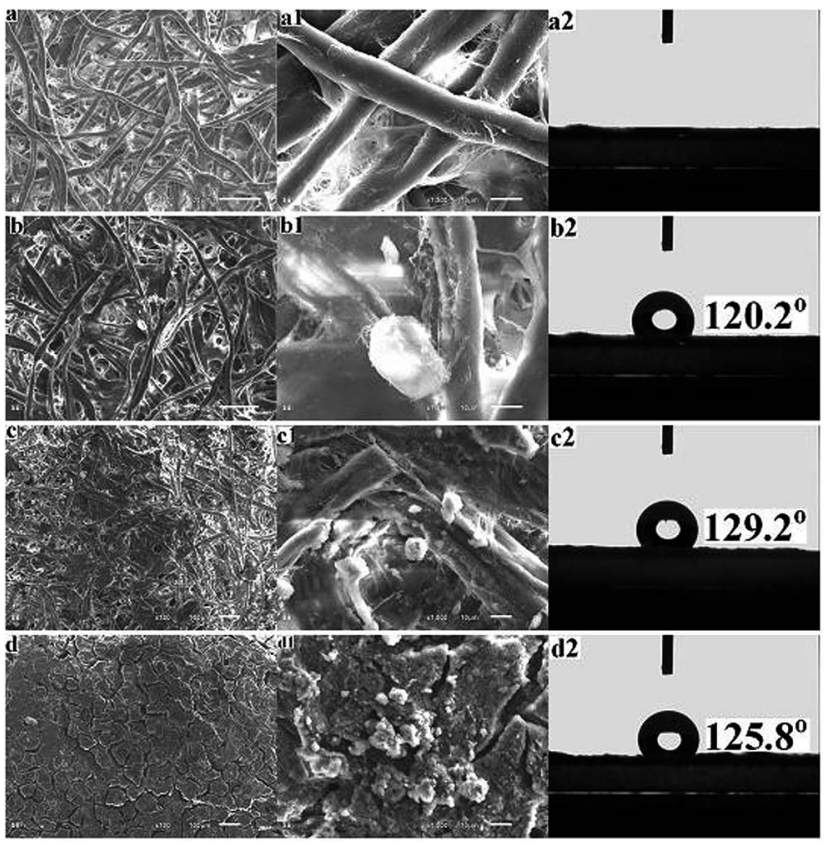

Fig. 1 SEM and WCA images of the original ( $a, a 1$, and $a 2)$, and modified filter paper by $2 \mathrm{wt} \%$ (b, b1 and b2), 3 wt\% (c, c1 and c2), and 4 wt\% (d, d1, and d2). Scale bar: $100 \mu \mathrm{m}$ for (a-d); $10 \mu \mathrm{m}$ for (a1-d1).

by dip-coating method. Considering that the wettability of surface is controlled by the chemical composition and the surface roughness, silica nanoparticles were dip-coated together with silane coupler to increase the surface hydrophobicity and roughness of filter paper. The morphologies of the original (Fig. 1a) and modified (Fig. 1b-d) filter paper were observed by SEM. In Fig. 1b and c, the cellulose fibers exhibit similar self-aggregation and highly porous microstructures to Fig. 1a, which demonstrates that there is no significant effect on the pore size and the permeability of filter paper during this procedure. However, with silica content increased to $4 \%$, cellulose fibers can be covered by a silica layer with cracks, easily resulting in falling away (Fig. 1d).

After modification, some polysiloxane particles with diameter ranging from hundreds of nanometers to several micrometers appeared on the surface of the modified filter paper as

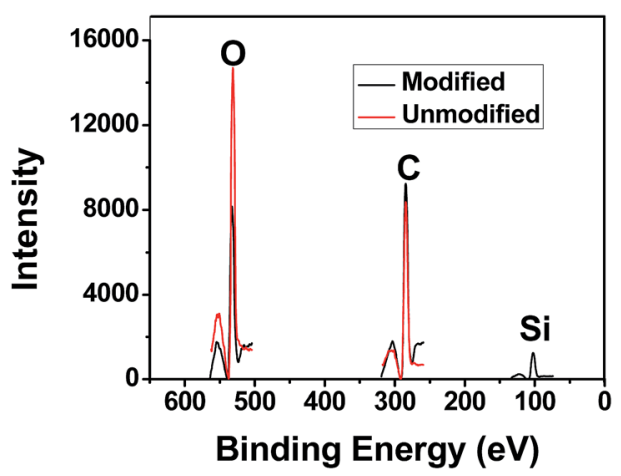

Fig. 2 XPS spectra on the surface of the filter paper before and after modification. 


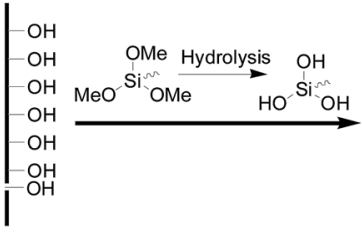

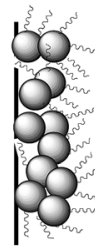

Silica Nanoparticle
Scheme 1 Interaction between cellulose, silica nanoparticles, and HDTMS.

shown in Fig. 1b-c. Much rougher surface indicates the formation of uneven silica coating. It can be estimated the coating thickness varying from $0.5 \mu \mathrm{m}$ to $5 \mu \mathrm{m}$ by SEM images. The element components on the surface of the filter paper before and after modification was analyzed by XPS (Fig. 2). Si element occurred as expected after modification, which confirms that HDTMS and silica nanoparticles have been successfully anchored onto the filter paper after modification.

The formation mechanism of the polysiloxane particles is schematically presented in Scheme 1 . The small amount of water in the solution led to the hydrolysis of HDTMS, then the obtained silanols would interact with the hydroxyl groups on the surface of filter paper and silica nanoparticles, and other silanols. The reaction between the silanols and the hydroxyl groups resulted in a covalently attached silane layer on the surface of the filter paper, and the formation of the polysiloxane particles can be attributed to the self-polymerization of silanols. The hierarchically micro-/nano-structure surface, along with the hydrophobicity of hexadecyl groups, results in the more hydrophobic coating.

\section{Wettability}

The wettability of the modified filter paper was characterized by water contact angle (WCA). The unmodified filter paper exhibited hydrophilic property, as the filter paper could absorb water drop rapidly. On the contrary, the modified filter paper were highly hydrophobic, exhibiting WCA above $120^{\circ}$ depending on the concentration of silica nanoparticle (Fig. 1b2, c2, and d2). The modified filter paper by $3 \mathrm{wt} \%$ silica solution showed a WCA up to around $130^{\circ}$ (Fig. 1c2). The surface wettability is ruled by the surface energy and roughness according to previous research. ${ }^{25-27}$ In this case, the surface energy of the modified filter paper decreased by silanization reaction, and the formation of polysiloxane particles endowed the modified filter paper with high surface roughness. ${ }^{1}$ The original filter paper instantaneously absorbed water and petroleum ether, indicating its amphiphilic property. Owing to its hydrophobicity and lipophilicity, the modified filter paper absorbed petroleum ether, while water drops remained on the surface, which demonstrated that it is potential candidate for oil-water separation.

\section{Separation of oil-water mixtures and emulsions}

For the original filter paper, a mixture of oil and deionized water (dyed with methylene blue) was poured into separation device, it can be seen that oil and water simultaneously flowed down, attributing its amphiphilicity. However, the mixture was poured into the device equipped with the modified filter paper, and the light oil first rapidly permeated through while water retained due to its hydrophobicity and lipophilicity.

To test the emulsion separation quality, SDS-stabilized oilwater emulsions were employed, in which the size of the drops varied from hundreds of nanometers to several micrometers. No demulsification or precipitation is observed in the emulsions for $24 \mathrm{~h}$ in ambient conditions, indicating their fine stability. During the emulsion separation process, petroleum ether completed through the modified filter paper, whereas water retained. As a result, the filtrate was clear and transparent without any color, while the retention also became uniform with blue color, indicating the petroleum ether-water emulsion could be separated with high quality. The separation of waterin-oil emulsions including water-in-dichloromethane and water-in-toluene was carried out in accordance with the same steps. As shown in Fig. 3, no droplets are observed in the collected filtrates. Furthermore, DLS measurements reveal the difference in droplet size before and after separation precisely. In general, superhydrophobic materials with WCA up to $150^{\circ}$
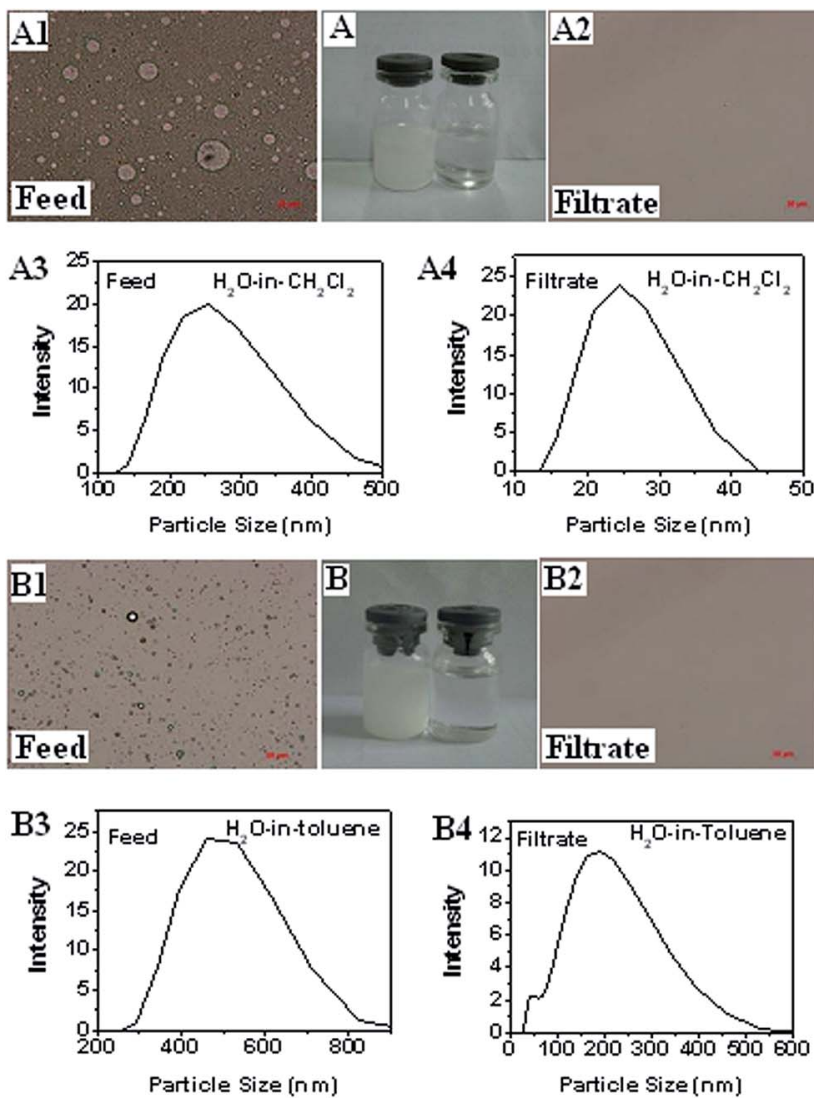

Fig. 3 Optical microscopy images of SDS-stabilized water-indichloromethane emulsion (A) and SDS-stabilized water-in-toluene emulsion (B) before (A1 and B1) and after (A2 and B2) filtration (scale bars: $50 \mu \mathrm{m}$ ); DLS results of droplet size before (A3 and B3) and after (A4 and B4) filtration of the SDS-stabilized water-in-dichloromethane emulsion and SDS-stabilized water-in-toluene emulsion by the modified filter paper. 
can be employed for oil-water separation, in this case, the modified filter paper with WCA around $130^{\circ}$ also possesses excellent separation property. This result demonstrates that superhydrophobicity is not an essential term for oil-water separation.

It is worthy of note that the entire separation process is driven solely by gravity without any other external force. The mixture and emulsion of petroleum and water could be separated successfully in one-step. The separation efficiency can be determined by calculating the mass ratio of selected filtrate to the feed. ${ }^{28}$ The modified filter paper exhibits high separation efficiency of $>98 \%$ for oil-water mixture and emulsion. In addition, the emulsion could be separated even if the size of the emulsion droplet is smaller than the pore size of modified filter paper due to the fact that the separation is based on coalescence separation rather than size sieving filtration, which intercepts the emulsified droplets by the coalescence of droplets once contacting with the fabric coatings. ${ }^{29,30}$ In the separation processes of water-in-oil emulsions, water droplets were captured on the modified surfaces, and the droplet volume increased upon coalescence with other water droplets in the oil phase. For water-in-dichloromethane system, the captured droplets eventually self-released from the surfaces under the influence of buoyancy and rose to the air-oil interface due to the higher density of dichloromethane. However, for waterin-toluene system, the captured droplet accumulated, and the modified surface were partially water-wettable, which enabled the adhesion but not the spreading of water droplet due to the lower density of toluene.

\section{Durability and reusability}

Considering that the filter paper is easily damaged, the durability were tested. The results showed that the durability is relative to the concentration of silica nanoparticles. For the sample modified by 2 wt $\%$ silica solution, no water permeated through the filter paper to the oil phase within 15 min after complete oil-water separation. However, a similar process lasted for over $24 \mathrm{~h}$ for that modified by $3 \mathrm{wt} \%$ silica solution. Oil-water separation states under different period and recycling times were shown in Fig. 4. All samples in Fig. 4A1 and A2 achieved complete oil-water separation within $15 \mathrm{~min}$, even after $3 \mathrm{~h}$, the samples still kept as their original states. After 5 repeatable separation cycles, for sample 1 and 2 (SDSstabilized oil-water emulsion), water leak occurred within 15 min (Fig. 4B1), however, sample 3 and 4 for oil-water mixture separation still kept as the original state even after $3 \mathrm{~h}$ (Fig. 4B2). After 10 repeatable cycles, sample 3 and 4 for oil-water mixture separation kept their original states.

The surface microstructure of the modified filter paper under different recycling times was shown in Fig. 5. It can be seen that, after 5 and 10 repeatable cycles, the filter paper for oil-water mixture separation kept the original state. However, the filter paper for oil-water emulsion separation exists cracks on the surface after 5 cycles, resulting in water leak, which is in accordance with the result in Fig. 4B1.

After 10 repeatable separation cycles of oil-water mixture, there is still a high separation efficiency of $98 \%$, indicating a good recyclability of the modified filter paper. With regard to

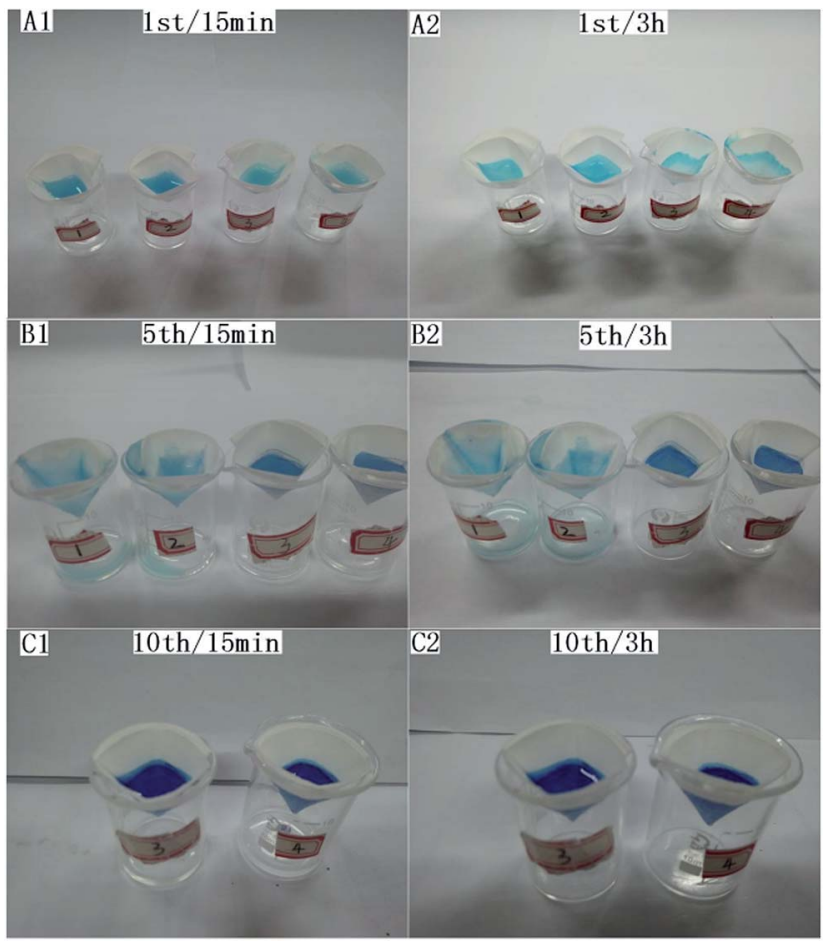

Fig. 4 Separation images of oil-water emulsion (sample 1 and 2) and mixture (sample 3 and 4) under different separation period (15 min and $3 \mathrm{~h}$ ) and recycling times (1, 5, and 10).

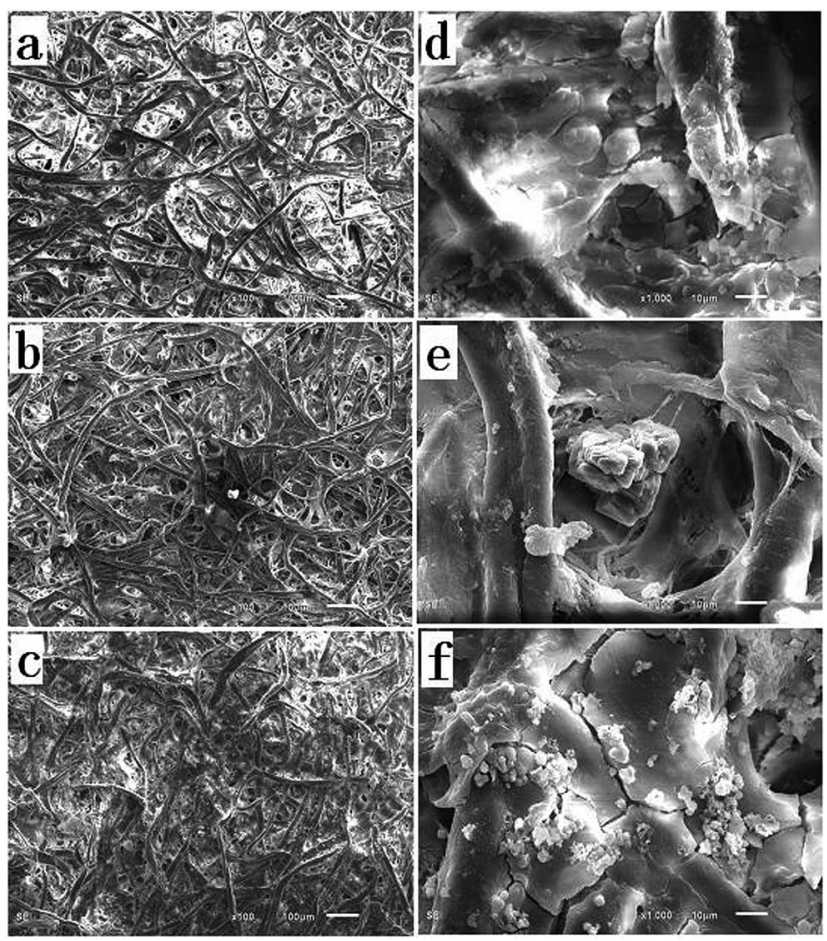

Fig. 5 SEM images of the modified filter paper under different recycling times ((a) and (d) 5 times by oil-water mixture; (b) and (e) 10 times by oil-water mixture; (c) and (f) 5 times by oil-water emulsion). Scale bar: $100 \mu \mathrm{m}$ for $(a-c) ; 10 \mu \mathrm{m}$ for $(d-f)$. 
the wettablility, WCA decreased from $130^{\circ}$ to around $125^{\circ}$ after 10 repeatable cycles, maintaining well hydrophobic property. The loss of hydrophobicity can be ascribed to the partial removal of polysiloxane particles at the filter paper during separation experiments. In addition, the waste filter paper can be treated by simple combustion.

\section{Conclusions}

We have demonstrated that oil-water filter paper can be prepared via a facile and almost one-step procedure. Owing to the successful introduction of polysiloxane particles on the surface during silanization process, the as-prepared displayed hydrophobic property and high efficiency for oil-water separation. The modified filter paper could not only selectively permeate oil from mixture, but also exhibit superior recyclability with over 10 times, indicating its great potential application. This facile, low cost, time-consuming saving, and environmentally friendly method will open up a new avenue to fabricate novel interfacial materials for oil-water separation.

\section{Acknowledgements}

The authors gratefully acknowledge financial support from Shandong Provincial Natural Science Foundation of China (Grant No. 2016ZRB01232), Shandong Provincial Science and Technology Development Plan Project of China (2014GGX102013), and Shandong Provincial Overseas Visiting Scholar Project of China (2011).

\section{Notes and references}

1 S. Zhou, P. Liu, M. Wang, H. Zhao, J. Yang and F. Xu, ACS Sustainable Chem. Eng., 2016, 4, 6409.

2 W. Zhang, N. Liu, Y. Cao, Y. Chen, Q. Zhang, X. Lin, R. Qu, H. Li and L. Feng, ACS Appl. Mater. Interfaces, 2016, 8, 21816.

3 R. V. Kumar, A. K. Ghoshal and G. Pugazhenthi, J. Membr. Sci., 2015, 490, 92.

4 Z. Xue, Y. Cao, N. Liu, L. Feng and L. Jiang, J. Mater. Chem. A, 2014, 2, 2445.

5 Z. Dang, L. Liu, Y. Li, Y. Xiang and G. Guo, ACS Appl. Mater. Interfaces, 2016, 8, 31281.

6 J. Shieh, F. J. Hou, Y. C. Chen, H. M. Chen, S. P. Yang, C. C. Cheng and H. L. Chen, Adv. Mater., 2010, 22, 597.

7 A. Balram, S. Santhanagopalan, B. Hao, Y. K. Yap and D. D. Meng, Adv. Funct. Mater., 2016, 26, 2571.
8 A. Checco, A. Rahman and C. T. Black, Adv. Mater., 2014, 26, 886.

9 J. Zhang and S. Seeger, Adv. Funct. Mater., 2011, 21, 4699.

10 H. S. Lim, j. h. Baek, K. Park, H. S. Shin and J. H. Cho, Adv. Mater., 2010, 22, 2138.

11 J. Li, R. Kang, X. Tang, H. She, Y. Yang and F. Zha, Nanoscale, 2016, 8, 7638.

12 J. Li, C. Xu, Y. Zhang, R. Wang, F. Zha and H. She, J. Mater. Chem. A, 2016, 4, 15546.

13 J. Li, L. Yan, H. Li, J. Li, F. Zha and Z. Lei, $R S C A d v .$, 2015, 5, 53802.

14 J. Li, L. Yan, X. Tang, H. Feng, D. Hu and F. Zha, Adv. Mater. Interfaces, 2016, 3, 1500770.

15 G. Wen, Z. Guo and W. Liu, Nanoscale, 2017, 9, 3338.

16 T. Darmanin and F. Guittard, J. Mater. Chem. A, 2014, 2, 16319.

17 Z. Shi, W. B. Zhang, F. Zhang, X. Liu, D. Wang, J. Jin and L. Jiang, Adv. Mater., 2013, 25, 2422.

18 J. Songok and M. Toivakka, ACS Appl. Mater. Interfaces, 2016, 8, 30523.

19 Y. Lin, D. Gritsenko, Q. Liu, X. Lu and J. Xu, ACS Appl. Mater. Interfaces, 2016, 8, 20501.

20 S. K. Mahadeva, K. Walus and B. Stoeber, ACS Appl. Mater. Interfaces, 2015, 7, 8345.

21 S. Wang, M. Li and Q. Lu, ACS Appl. Mater. Interfaces, 2010, 2, 677.

22 J. B. Fan, Y. Song, S. Wang, J. Meng, G. Yang, X. Guo, L. Feng and L. Jiang, Adv. Funct. Mater., 2015, 25, 5368.

23 H. Ogihara, J. Xie, J. Okagaki and T. Saji, Langmuir, 2012, 28, 4605.

24 X. Tang, S. Nan, T. Wang, Y. Chen, F. Yu, G. Zhang and M. Pei, RSC Adv., 2013, 3, 15571.

25 L. Feng, Z. Y. Zhang, Z. H. Mai, Y. M. Ma, B. Q. Liu, L. Jiang and D. B. Zhu, Angew. Chem., Int. Ed., 2004, 43, 2012.

26 Y. Si and Z. Guo, Nanoscale, 2015, 7, 5922.

27 Y. Yang, H. Yi and C. Wang, ACS Sustainable Chem. Eng., 2015, 3, 3012.

28 Z. J. Wang, Y. Wang and G. J. Liu, Angew. Chem., Int. Ed., 2016, 55, 1291.

29 Y. Si, Q. X. Fu, X. Q. Wang, J. Zhu, J. Y. Yu, G. Sun and B. Ding, ACS Nano, 2015, 9, 3791.

30 K. Y. Tan, T. L. Hughes, M. Nagl and W. T. S. Huck, ACS Appl. Mater. Interfaces, 2012, 4, 6403. 